\title{
E2F1 acts as a negative feedback regulator of c-Myc-induced hTERT transcription during tumorigenesis
}

\author{
YAFEI ZHANG ${ }^{1,2}$, ANRAN ZHANG $^{1}$, CAIFEI SHEN $^{1}$, BICHENG ZHANG $^{3}$, ZHIGUO RAO $^{3}$, \\ RONGQUAN WANG ${ }^{1}$, SHIMING YANG ${ }^{4}$, SHOUBIN NING ${ }^{2}$, GAOPING MAO $^{2}$ and DIANCHUN FANG ${ }^{1}$ \\ ${ }^{1}$ Department of Gastroenterology, Southwest Hospital, Third Military Medical University, Chongqing; \\ ${ }^{2}$ Department of Gastroenterology, Air Force General Hospital of Chinese PLA, Beijing; \\ ${ }^{3}$ Department of Oncology, Wuhan General Hospital of Guangzhou Command, People's Liberation Army, Wuhan; \\ ${ }^{4}$ Department of Gastroenterology, Xinqiao Hospital, Third Military Medical University, Chongqing, P.R. China
}

Received April 9, 2014; Accepted June 3, 2014

DOI: $10.3892 /$ or.2014.3287

\begin{abstract}
Since induction of hTERT expression and subsequent telomerase activation play a critical role in the multistep process of tumorigenesis, a better understanding of hTERT regulation may provide not only a rationale for the molecular basis of cancer progression but also a path to the development of cancer prevention. The c-Myc oncoprotein can function effectively in activating the transcriptional expression of hTERT through E-box elements on its promoter. E2F transcription factor 1 (E2F1) was found to be a repressor of hTERT transcription by directly binding to its promoter, thereby inhibiting hTERT protein expression. For the extensively crosstalk between c-Myc and E2F1 signals, which is now known to be vital to cell fate, we speculated that E2F1 may play a negative regulatory role in c-Myc-induced hTERT transcription. In the present study, we chose to use human embryonic fibroblast cells as an experimental model system, and present evidence that the E2F1 transcription factor constitutes a negative regulatory system to limit c-Myc transcriptional activation of hTERT in normal cells. Furthermore, we demonstrated that upregulation of the miR-17-92 cluster (miR-20a/miR-17-5p) is involved in the regulation of E2F1-mediated negative feedback of the c-Myc/hTERT pathway. Our results not only reveal novel insights into how normal cells control the transmission of c-Myc-mediated oncogenic signals, but also further establish E2F1 as an important molecular target for cancer therapy.
\end{abstract}

Correspondence to: Professor Dianchun Fang, Department of Gastroenterology, Southwest Hospital, Third Military Medical University, Chongqing, P.R. China

E-mail: fangdianchun@hotmail.com

Professor Gaoping Mao, Department of Gastroenterology, Air Force General Hospital of Chinese PLA, Beijing, P.R. China

E-mail: maogaoping2012@163.com

Key words: E2F1, c-Myc, hTERT, miR-17-92 cluster, negative feedback regulation

\section{Introduction}

Telomere maintenance by telomerase has been proposed as an essential prerequisite for cell immortalization and tumorigenesis. Human telomerase reverse transcriptase, hTERT, is the catalytic subunit of telomerase, and its expression has been proven to be the rate-limiting step for telomerase activity (1). Studies have revealed that hTERT is expressed in over $85 \%$ of human tumors, whereas most normal human somatic tissues exhibit little or no expression $(2,3)$. The differential hTERT expression between tumor tissues and healthy normal tissues makes this gene a reasonable candidate for association analysis of tumorigenesis.

The c-Myc proto-oncogene plays an important role in the regulation of many cellular programs, including proliferation, differentiation, self-renewal and apoptosis. Aberrant expression of c-Myc is believed to be closely associated with cell immortalization and tumorigenesis (4). Thirty years ago, when c-Myc was initially classified as an oncogenic protein that cooperates with Ras to transform primary embryonic rat cells, it was already recognized that c-Myc activation contributes to cell immortalization (5). Consistent with this, some years later hTERT was identified as a direct transcriptional target of c-Myc, and the transcriptional induction is independent of additional protein synthesis $(6,7)$. These findings define a pathway of cell immortalization in which oncogenic c-Myc activation induces expression of hTERT, and enhanced hTERT expression leads to cell immortalization.

Evidence from numerous studies has clearly shown that c-Myc-dependent hTERT transcription is one of the major mechanisms for maintained expression of telomerase and apparently contributes to tumorigenesis. However, not all incidences of increased c-Myc expression in normal cells will result in activation of oncogenic sequences including hTERT (8). E2F transcription factor 1 (E2F1) is a member of the E2F family of transcription factors and displays multiple activities that could be involved in cell differentiation, development and apoptosis. E2F1 was determined to be transcriptional activated by c-Myc, and has been shown to crosstalk extensively with c-Myc signaling, which is now believed to control cell fate decisions $(9,10)$. Of special interest to us, as a transcriptional 
regulator, E2F1 was found to be able to inhibit hTERT gene transcription (11-13). Therefore, we speculated that E2F1 may be a potential suppressor of c-Myc-induced hTERT activation, which exhibits a negative feedback regulation in normal cells upon c-Myc oncogenic signals.

The miR-17-92 gene cluster is frequently overexpressed in human cancers, and has been shown to promote several aspects of oncogenic transformation (14). Recent evidence has demonstrated that E2F1 is inhibited at the post-transcriptional step by members of miR-17-92 (miR-20a and miR-17-5p) (15-17). However, both E2F and Myc can induce the transcription of miR-17-92, thus forming a complicated negative feedback system in the interaction network (18-20). At present, some scholars believe that miR-17-92 represents a molecular switch for cell proliferation and apoptosis via determining the levels of these two proteins $(21,22)$. Based on these findings, we speculated that miR-17-92 plays a critical mediating role in the expression of hTERT via regulating c-Myc/E2F1 networks.

In this study, we chose to use human embryonic fibroblast (HEF) cells as the model system, and performed experiments to investigate whether E2F1 can act as a negative feedback regulator of hTERT transcription in response to c-Myc activation. Furthermore, we analyzed the effects of the upregulation of miR-17-92 (miR-20a and miR-17-5p) expression on the crosstalk of c-Myc/E2F1 and downstream hTERT expression.

\section{Materials and methods}

Cell culture and reagents. The HEF cells (passage 10) used in this study have been described previously (23). Cells were cultured in DMEM supplemented with 10\% FCS (Invitrogen Corp., Carlsbad, CA, USA), at $37^{\circ} \mathrm{C}$ in a humidified atmosphere containing $5 \% \mathrm{CO}_{2}$. An adenovirus (type 5) expressing c-Myc (rAd-c-Myc) was produced by subcloning full-length c-Myc into the vector pShuttle, and the recombinant adenovirus was produced by overlap recombination. The HA-E2F1 plasmid and hTERT promoter plasmid were constructed by standard genetic manipulations. The mutant hTERT promoter construct was generated by the Site-Directed Mutagenesis kit (Stratagene, Santa Clara, CA, USA) in accordance with the manufacturer's standard procedure using the wild-type hTERT promoter construct as a template. The antisense oligonucleotide directed to E2F1 mRNA (5'-TAGATC CGATCCAGCTCAGTGACA-3') was designed and provided by Sangon Biological Co., Shanghai, China. The full-length backbone was modified to avoid enzymatic cleavage by DNases.

Quantitative RT-PCR analysis. For quantitative realtime PCR (qRT-PCR) analysis, total-RNA was extracted with TRIzol (Takara, Dalian, China), and cDNA was prepared using the PrimerScript reverse transcriptase kit (Takara). The mRNA expression levels of the targeted genes were analyzed by qRT-PCR using Applied Biosystems 7500 Real-Time PCR and SYBR-GreenER ${ }^{\mathrm{TM}}$ reagent system from Invitrogen. The primers used in this study are listed as following: c-Myc, F: 5'-TCACCAGCACAACTACGCCG-3' and R: 5'-CAGG ATGTAGGCGGTGGCTT-3'; E2F1, F: 5'-AGGGGTGTGG GGTTGATACC-3' and R: 5'-TCAGACACTGCAGGAGG
GAC-3'; hTERT, F: 5'-CGGAAGAGTGTCTGGAGCAA-3' and R: 5'-GGATGAAGCGGAGTCTGGA-3'. Expression of the reference $\beta$-actin gene was used to normalize the expression of the mRNA for each targeted gene. Data were analyzed using the $2-{ }^{\Delta \Delta \mathrm{Ct}}$ method.

Western blot analysis. Total cell lysates were prepared in $1 \mathrm{X}$ SDS buffer. Proteins were separated by SDS-PAGE and transferred onto PVDF membranes. Membranes were then blotted with monoclonal antibodies specific to c-Myc (Santa Cruz Biotechnology, Santa Cruz, CA, USA), E2F1 (Abcam, Cambridge, MA, USA), hTERT (Santa Cruz) and $\beta$-actin (Santa Cruz). Antigen-antibody complexes were visualized with enhanced chemiluminescence (Thermo Scientific, Rockford, IL, USA).

Telomerase assay. To quantitatively detect changes in telomerase levels, cells were assayed for telomerase activity using the telomerase polymerase chain reaction-enzymelinked immunosorbent assay (PCR-ELISA) (Roche Diagnostics, Mannheim, Germany) according to the manufacturer's directions and as performed previously (24). Briefly, cells were washed with Dulbecco's phosphate-buffered saline, lysed in CHAPS lysis buffer and then assayed for protein using the Bio-Rad DC protein assay (Bio-Rad, Hercules, CA, USA) according to the manufacturer's instructions. Cell extracts equivalent to $3 \mathrm{mg}$ of protein were used. Following PCR-ELISA, telomerase activity was detected using a Dynex-MRX plate reader and recorded as absorbance units.

Apoptosis analysis. Detection of apoptotic cells by flow cytometry was performed as described previously (25). HEF cells cultured in 6-well plates were infected with recombinant adenoviruses at various MOIs for $48 \mathrm{~h}$ before cells were harvested, and then the Annexin V/propidium iodide binding assay was performed using a flow cytometer (XL-MCL; Coulter Epics, Miami, FL, USA).

Reporter assay. HEF cells were transfected with the indicated plasmids using Lipofectamine 2000 (Invitrogen) for $48 \mathrm{~h}$, and the luciferase activity was measured using the dual luciferase system (Promega, Madison, WI, USA) as described previously (26). The relative luciferase activity was expressed as a ratio of the firefly luciferase activity to the Renilla luciferase activity.

ChIP and ChIP-re-ChIP assays. Chromatin immunoprecipitation (ChIP) experiments were performed using a ChIP assay kit (Upstate Biotechnology, Millipore, CA, USA) according to the manufacturer's instructions. Cross-linked proteins were immunoprecipitated using an anti-E2F1 (Abcam), and normal mouse IgG (Santa Cruz). For ChIP-re-ChIP experiments, primary immunocomplexes obtained with anti-E2F1 or anti-c-Myc were washed and eluted with $10 \mathrm{mM}$ dithiothreitol, and eluates were diluted in IP dilution buffer to perform re-ChIP with antic-Myc or anti-E2F1. The recovered DNA was amplified with specific primer pairs and analyzed by qRT-PCR. Normal IgG and input DNA values were used to subtract and normalize values from ChIP and ChIP-re-ChIP samples. The percent 


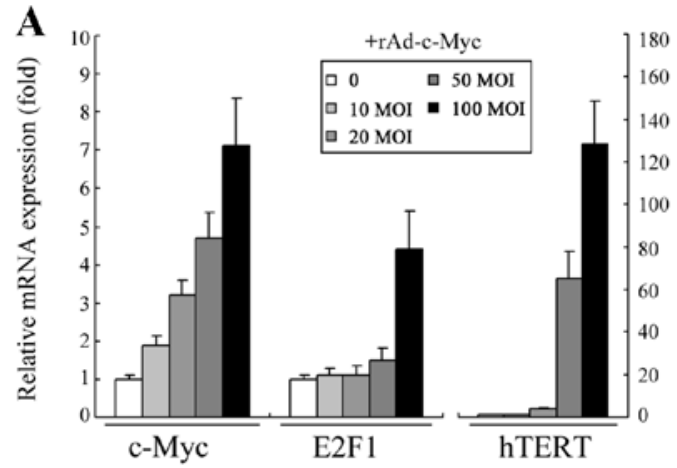

C

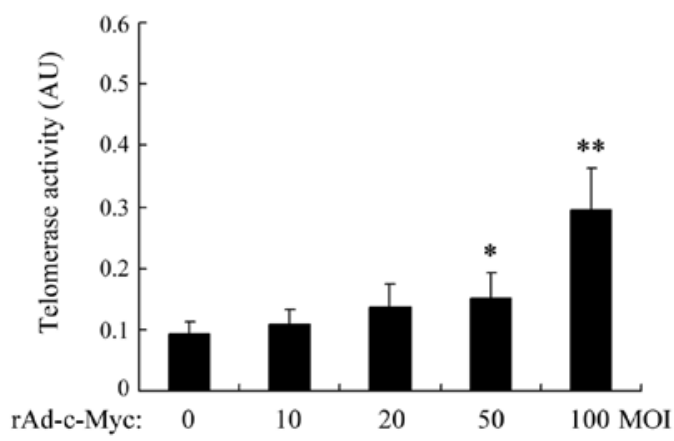

B

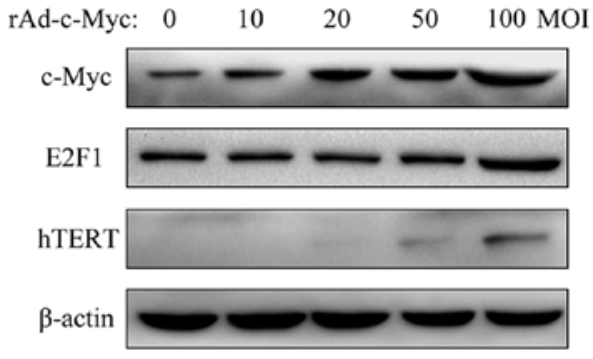

D

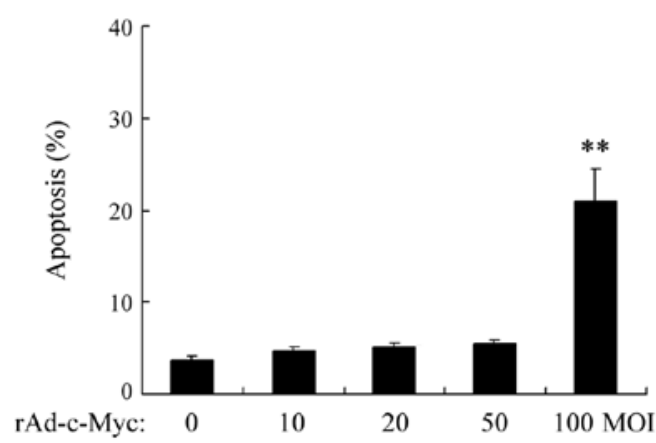

Figure 1. E2F1 is associated with hTERT expression and telomerase activity in c-Myc-transfected HEF cells. Cells were transfected with rAd-c-Myc (MOI 0, 10, 20, 50 and 100) for $48 \mathrm{~h}$. (A) The mRNA expression of c-Myc, E2F1 and hTERT was assessed by qRT-PCR. The histogram summarizes the results from three independent experiments. (B) The protein expression of c-Myc, E2F1 and hTERT was assessed by western blot analysis. Representative results from three independent experiments with similar results are shown. (C) Telomerase activity was assessed by TRAP-PCR-ELISA assay. (D) The percentage of apoptotic cells after c-Myc transfection was analyzed using Annexin/PI staining. Values are means \pm SD of five independent experiments. ${ }^{*}<0.05$ and ${ }^{* *} \mathrm{P}<0.01$, vs. the blank control.

input method was used to quantitate the values of the immunoprecipitated DNA. Primers used for the hTERT promoter region were as follows: forward, 5'-GAGCAGCTGCGCTGT-3' and reverse, 5'-AGCTGGAAGGTGAAG-3'.

miRNA expression level assays. The expression levels of mature miRNAs were measured by TaqMan MicroRNA assays kit (Applied Biosystems, Foster City, CA, USA) following the manufacturer's protocol. The U6 primers were used as a normalization control. For relative expression levels, the $2^{-\Delta \Delta \mathrm{Ct}}$ method was used as previously described (27). Experiments were carried out in triplicate for each data point, and data analysis was carried out using Bio-Rad IQ software.

Statistical analysis. The data are expressed as means \pm SD. Differences were compared by one-way ANOVA analysis followed by LSD t-test. All statistical analyses were performed using SPSS 17.0 software (SPSS Inc., Chicago, IL, USA). $\mathrm{P}<0.05$ was considered to indicate a statistically significant difference.

\section{Results}

Co-expression of E2F1 increases the apoptosis of HEF cells following regulation of $c-M y c$-dependent hTERT induction. Both E2F1 and hTERT have been determined to be the direct transcriptional targets of c-Myc $(6,7,9,10)$. In this study, we initially verified whether c-Myc activation was sufficient to induce expression of both E2F1 and hTERT in the HEF cells. As illustrated in Fig. 1A and B, an increase in c-Myc activity by rAd-c-Myc transfection enhanced the expression of both E2F1 and hTERT at the mRNA and protein levels. However, intriguingly, no dose-dependent effect on E2F1 expression was observed. Upon severe c-Myc activation, significant E2F1 upregulation was induced, whereas following mild and moderate c-Myc activation, no significant E2F1 upregulation was noted. Regarding hTERT, an increase in the expression level was observed in a dose-dependent manner but was restricted to a limited amount even when c-Myc activity was greatly increased. We further tested the telomerase activity of HEF cells upon c-Myc activation. As shown in Fig. 1C, only moderate/high c-Myc inputs could result in a significant increase in telomerase activity. Since it has been determined that in normal cells elevated E2F1 expression participates in many aspects of the apoptotic process, we further detected the apoptosis of HEF cells. As shown in Fig. 1D, in the presence of high levels of c-Myc activation, the apoptosis rate of HEF cells was also significantly increased.

Specific inhibition of E2F1 expression leads to elevated $c$-Myc-induced hTERT transcription. Although the expression of hTERT after c-Myc activation in HEF cells was observed to be dose-dependent, it was restricted to a low level, and when hTERT expression was markedly increased, it was accompanied by an increased E2F1 expression of a magnitude that could induce significant apoptosis, suggesting a negative 
A

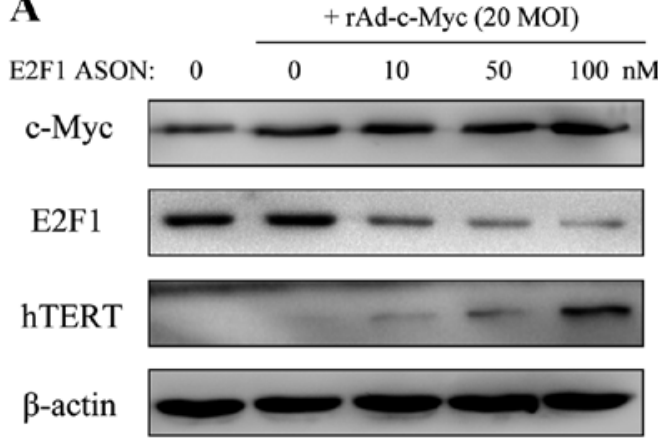

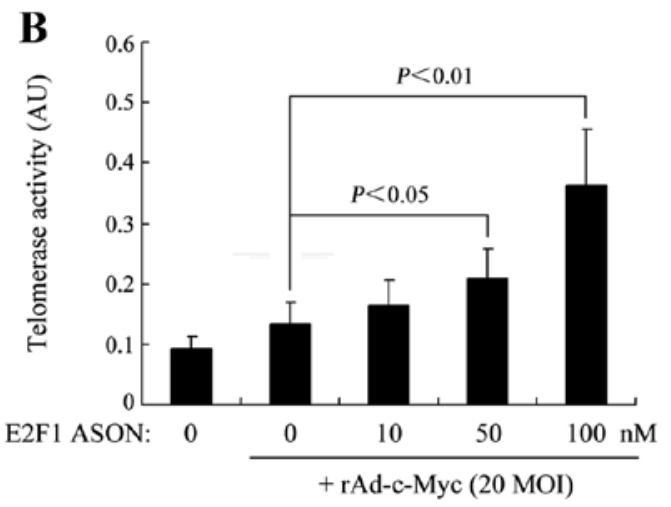

$\mathbf{E}$

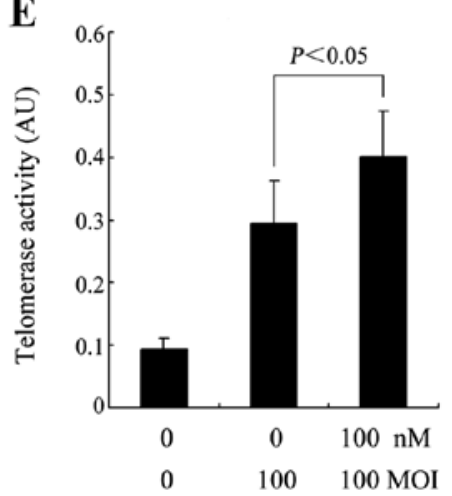

Figure 2. Inhibition of E2F1 enhancs hTERT expression and telomerase activity. (A and B) HEF cells were simultaneously transfected with 20 MOI rAd-cMyc and with various concentrations of E2F1-specific antisense oligonucleotide (ASON) for 48 h. Target proteins were detected by western blot analysis. Telomerase activity was assessed by TRAP-PCR-ELISA assay. (C-E) HEF cells were simultaneously transfected with 100 MOI rAd-c-Myc and 100 nM E2F1-specific ASON. Target proteins were detected by western blot analysis. Apoptosis and telomerase activity were assessed by Annexin/PI staining and TRAP-PCR-ELISA assay, respectively. Values are means \pm SD of five independent experiments.

feedback loop restricting hTERT activity during c-Myc oncogenesis. Therefore, we subsequently examined whether E2F1 inhibition has an effect on c-Myc-induced hTERT expression. As shown in Fig. 2A, a decrease in E2F1 expression by specific antisense oligonucleotide (ASON) at concentrations of 50 and $100 \mathrm{nM}$ led to a significant increase in hTERT expression after moderate c-Myc activation (20 MOI rAd-c-Myc), while as shown in Fig. 2B, the telomerase activity of HEF cells was also significantly increased. In addition, the enhanced apoptosis of HEF cells in response to a high level c-Myc activation (100 MOI rAd-c-Myc) was inhibited after specific blockage of E2F1 expression, while the increased telomerase activity of $\mathrm{HEF}$ cells was further enhanced by inhibiting E2F1 expression (Fig. 2C-E).

E2F1 is a transcriptional repressor for hTERT gene expression induced by c-Myc activation. E2F1 has been found to be able to bind the hTERT promoter (at -174 and $-98 \mathrm{bp}$ ) and repress its expression in several human cell lines (11-13). We therefore hypothesized that in normal cells E2F1 may act as a transcriptional repressor that restricts hTERT gene expression in response to oncogenic c-Myc stimulation. To test this hypothesis, we performed a luciferase reporter assay to examine whether E2F1 regulates hTERT promoter activity in HEF cells and found that overexpression of E2F1 significantly reduced the hTERT promoter activity (Fig. 3A and B). To detect the direct binding of E2F1 to the hTERT promoter, we next performed ChIP assay using the anti-E2F1 antibody in HEF cells transfected with 20 MOI rAd-c-Myc. The qRT-PCR results indicated that E2F1 could directly bind to the hTERT promoter region (Fig. 3C and D). Furthermore, when E2F1 expression was inhibited by a specific ASON, the qRT-PCR expression levels of the immunoprecipitated hTERT promoter region was observed to have a measurable reduction (Fig. 3D). Finally, to demonstrate that E2F1 controls c-Myc-induced hTERT gene expression, we performed ChIP-re-ChIP assay in rAd-c-Myc-transfected HEF cells by using anti-E2F1 and anti-c-Myc antibodies. As shown in Fig. 3E, the qRT-PCR data indicated that E2F1 and c-Myc simultaneously associated on the hTERT promoter region.

Overexpression of the miR-17-92 cluster attenuates the E2F1dependent inhibition of hTERT in c-Myc-activated HEF cells. Considerable evidence indicates that members of the miR-17-92 cluster, miR-20a and miR-17-5p, play critical roles in the regulation of c-Myc/E2F1 networks (15-22). In this study, we further investigated the effect of $\mathrm{miR}-20 \mathrm{a} / \mathrm{miR}-17-5 \mathrm{p}$ overexpression on E2F1-dependent inhibition of hTERT in c-Myc-activated HEF cells. To ascertain whether miR-20a/miR-17-5p directly regulate E2F1 expression through the target sites in the 3'UTR of E2F1 mRNA, we constructed a luciferase reporter vector with the two putative E2F1 3'UTR target sites for miR-20a/ miR-17-5p downstream of the luciferase gene (pMIRE2F1-3'UTR) and the mutant version with a mutation of $6 \mathrm{bp}$ 
A
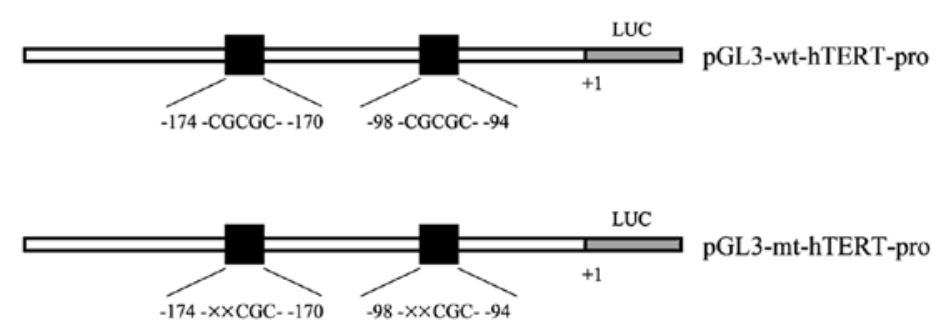

C

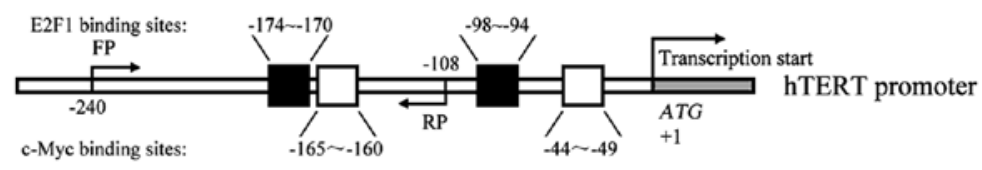

B

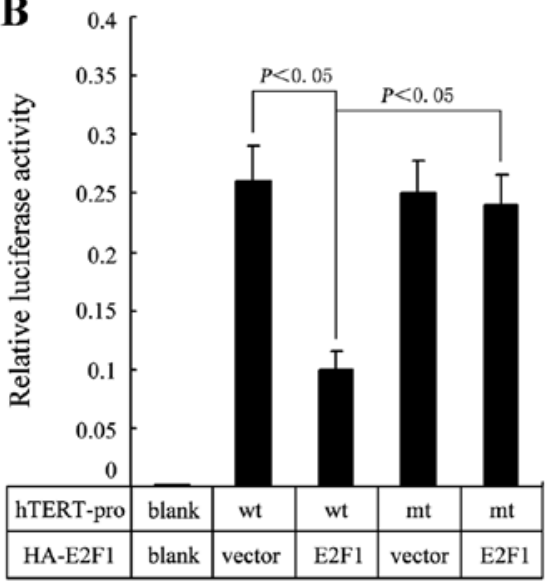

HA

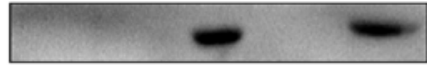

$\beta$-actin
D

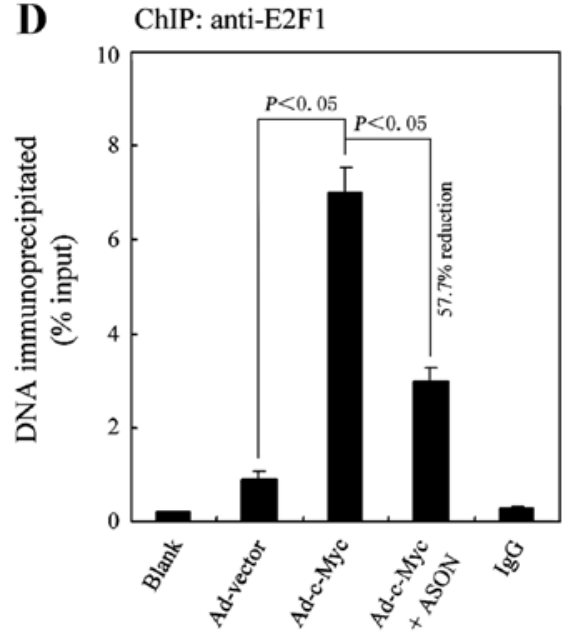

$\mathbf{E}$

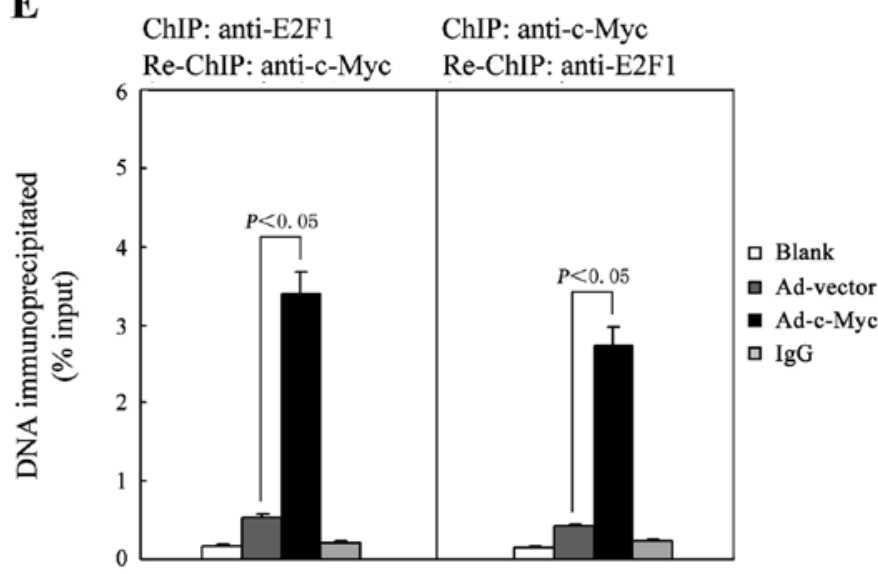

Figure 3. E2F1 is a repressor of hTERT gene transcriptional expression induced by c-Myc. (A) Schematic diagram of pGL3 promoter constructs carrying the wild-type sequence (PGL3-wt-hTERT) or the -98, 99/-174, 173 CG deletion (PGL3-mt-hTERT). (B) HEF cells were transfected with the indicated plasmids for $48 \mathrm{~h}$ and harvested for the hTERT reporter assay. The relative luciferase activity was presented as a ratio of the firefly luciferase activity to the Renilla luciferase activity and expressed as means $\pm S D(n=5)$. (C) Schematic diagram denoting the PCR primers used in the ChIP assays. FP, forward primer; RP, reverse primer. (D) The occupancy by E2F1 at the hTERT proximal promoter region in c-Myc-transfected HEF cells was assessed by ChIP assay. (E) ChIP-re-ChIP confirmed the association of E2F1 and c-Myc with the hTERT promoter. Antibodies used for the first and second ChIP are indicated above the lanes. The values were subtracted from the results for ChIP and ChIP-re-ChIP samples. Results represent the means \pm SD of five independent immunoprecipitations.

from the site of perfect complementarity (pMIR-mutant-E2F13'UTR) (Fig. 4A). Luciferase reporter vector together with the $\mathrm{miR}-20 \mathrm{a} / \mathrm{miR}-17-5 \mathrm{p}$ mimics or mimic control were transfected into the HEF cells. As shown in Fig. 4B, a significant decrease in relative luciferase activity was noted when pMIR-E2F13'UTR was co-transfected with miR-20a/miR-17-5p mimics, but not in the controls $(\mathrm{P}<0.05)$. In addition, we co-transfected HEF cells with 20 MOI rAd-c-Myc and miR-20a/miR-17-5p mimics. As shown in Fig. 4C, the expression levels of miR-20a and miR-17-5p were expected to be greatly increased after transfection. The expression levels of c-Myc, E2F1 and hTERT were detected by western blot analysis. As indicated in Fig. 4D, the protein expression of E2F1 was greatly suppressed in the HEF cells transfected with miR-20a/miR-17-5p mimics, while the expression of hTERT was greatly increased, accompanied by a significant increase in telomerase activity (Fig. 4E).

\section{Discussion}

Numerous studies indicate that there is extensive crosstalk between E2F1 and c-Myc signal transduction pathways, and this interaction is vital to cell viability and function. Rounbehler et al using K5 c-Myc-transgenic mice, showed that inactivation of E2F1 not only increased the incidence of tumor formation, but also promoted tumor initiation, indicating that E2F1 activation is a control mechanism serving to protect normal cells from c-Myc-induced tumorigenesis $(28,29)$. Observations that support this hypothesis also 
A

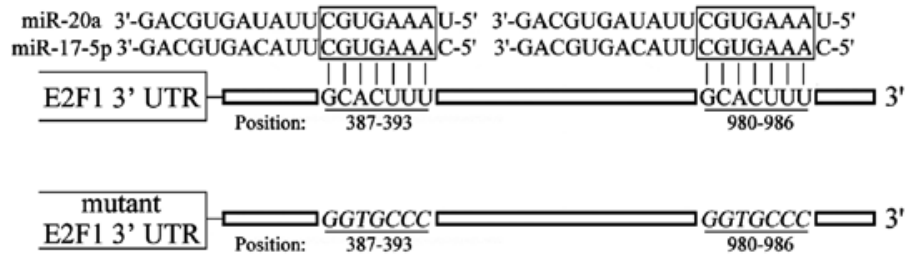

C

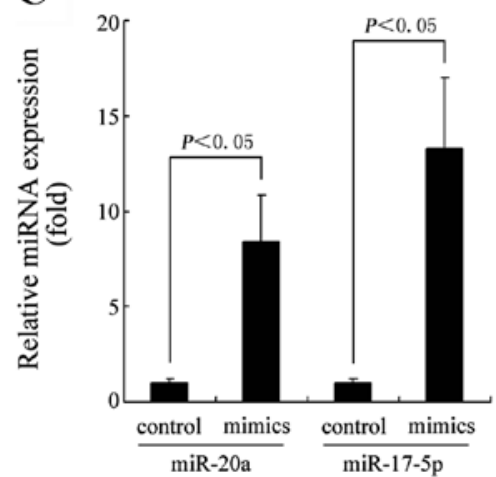

D

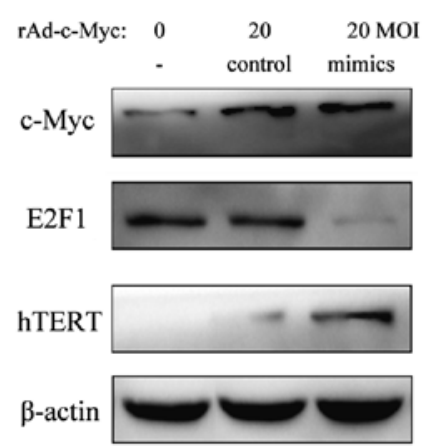

B

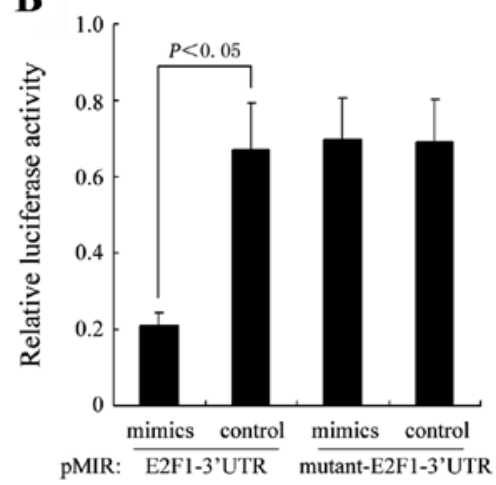

E

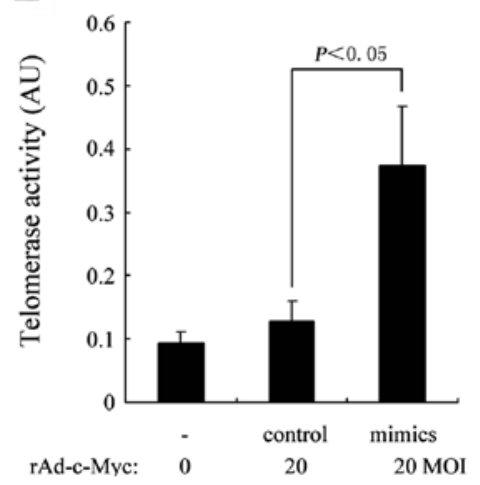

Figure 4. miR-20a/miR-17-5p are involved in the E2F1-mediated negative feedback regulation of c-Myc-induced hTERT transcription. (A) Schematic diagram of the predicted miR-20a/miR-17-5p binding sites of the E2F1 3'UTR. The bottom panel shows the site-mutated sequence. (B) Assay for luciferase activity was performed in HEF cells to determine the direct regulation of E2F1 by miR-20a/miR-17-5p. (C) The relative expression levels of miR-20a and miR-17-5p were measured by qRT-PCR and expressed as a fold-change compared with the mimic control. (D) The protein expression of c-Myc, E2F1 and hTERT was detected by western blot analysis. Representative results from three independent experiments with similar results are shown. (E) Telomerase activity of HEF cells after co-transfection with 20 MOI rAd-c-Myc and miR-20a/miR-17-5p mimics was assessed by TRAP-PCR-ELISA assay. Values are means \pm SD of five independent experiments.

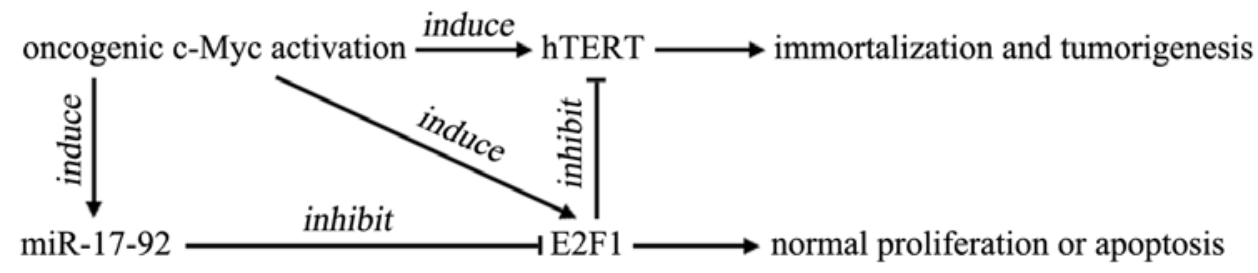

Figure 5. Schematic diagram of E2F1 and miR-17-92 involving c-Myc-induced hTERT transcription.

include the finding that i) c-Myc-mediated proliferation and lymphomagenesis are compromised by E2F1 loss (30) and ii) E2F1 blocks and c-Myc accelerates hepatic ploidy and tumorigenesis in transgenic mouse models (31). These results further indicate that $\mathrm{E} 2 \mathrm{~F} 1$ can counteract c-Myc oncogenic activation, especially in the process of tumor initiation.

It is recognized that $\mathrm{E} 2 \mathrm{~F} 1$ has a dual function: it triggers both proliferation and apoptosis via activation of downstream target genes (32). Overexpression of E2F1 causes apoptosis via p53-dependent and p53-independent pathways, and this is an effective mechanism for suppressing tumorigenesis $(33,34)$. In this study, we observed that high expression of the c-Myc oncoprotein induced significant apoptosis of HEF cells, and when E2F1 expression was specifically blocked, the enhanced apoptosis was markedly attenuated, suggesting the involvement of E2F1-mediated apoptosis. Of note, Leone et al revealed that $\mathrm{E} 2 \mathrm{~F} 1$ is required for c-Myc-induced apoptosis in mouse embryonic fibroblast cells (35). However, Rounbehler et al reported that in E2F1-null transgenic mice, tumorigenesis was accelerated while the c-Myc-induced apoptosis and proliferation were unaffected by the status of E2F1, indicating that E2F1 has other functions with which to suppress the oncogenic transformation by c-Myc $(28,29)$.

Induction of hTERT expression and subsequent telomerase activation constitute a critical step in tumorigenesis, especially in the process of tumor initiation (4). Studies have shown that c-Myc plays a critical role in the induction of hTERT gene transcription through the E-box located within the core promoter region (at -165 and $-49 \mathrm{bp})(6,7)$. However, in normal cells, not all c-Myc upregulation leads to the expression of hTERT 
and subsequent telomerase activation (8). In addition, studies have found that in normal cells including embryonic fibroblast cells, c-Myc induction is a common event which is required for the cellular response to various types of stress but detectable hTERT protein expression is very rare (36-38), indicating that the c-Myc-induced hTERT transcription is tightly controlled and limited in non-oncogenic cells.

Although E2F1 has been proven to be a suppressing regulator of hTERT expression, little is known concerning the influence of E2F1 on c-Myc-induced hTERT activation. In this study, we present evidence that E2F1 negatively regulates c-Myc-dependent hTERT transcription in HEF cells through direct binding to the promoter region. As E2F1 is also a transcriptional target of $\mathrm{c}-\mathrm{Myc}$, it thus establishes a negative feedback pathway in which responsive E2F1 protein expression inhibits hTERT expression and acts as an apoptotic trigger, thus counteracting the c-Myc oncogenic signal transduction in normal cells. Of note, we performed the same experiments in hTERT-negative human osteosarcoma U2OS cells but no expression changes of hTERT and E2F1 were observed upon rAd-c-Myc transfection (data not shown), indicating that the negative feedback pathway for suppression of hTERT transcription is no longer functional when tumorigenesis has occurred. Our study therefore extends the explanation of how E2F1 counteracts c-Myc oncogenic activation, and provides a clue to the understanding of mechanisms of hTERT activation in the process of tumor initiation.

The miR-17-92 cluster, also called oncomir-1, is among the first miRNAs to be validated as showing oncogenic potential. Genomic amplification and elevated expression of miR-17-92 were both found in various human malignances, and its enforced expression exhibits strong tumorigenic activity in multiple mouse tumor models (39). miR-17-92 carries out pleiotropic functions during both normal development and malignant transformation, as it acts to promote proliferation, inhibit differentiation, increase angiogenesis, and sustain cell survival (39). Considerable evidence indicates that the miR-17-92 cluster is critically involved in the regulation of c-Myc/E2F1 networks (15-22). E2F1 was found to be inhibited at the post-transcriptional level by members of the miR-17-92 cluster (miR-20a and miR-17-5p), while as transcription factors both c-Myc and E2F1 can induce the expression of microRNAs within the miR-17-92 cluster. Since both miR-17-92 and E2F1 can be transcriptionally activated by c-Myc, this establishes an unusual network in which c-Myc activates the transcription of E2F1 while simultaneously inhibiting its translation. Over the past few years, based on observations using transgenic mouse models and mathematical models, the regulation of miR-17-92 on c-Myc/E2F1 networks was thought to represent a molecular switch for cell proliferation and apoptosis. Therefore, we speculated that miR-17-92 also plays a regulatory role in the expression of hTERT via determining the relative protein level of c-Myc and E2F1. In this study, by performing a dual-luciferase reporter assay, we determined that in non-oncogenic HEF cells miR-17-92 (miR-20a/miR-17-5p) can directly inhibit E2F1 expression through the target sites in the 3'UTR of E2F1 mRNA. Furthermore, we investigated the effects of the upregulation of miR-20a/miR-17-5p expression on hTERT transcription in HEF cells with moderated c-Myc activation, and observed that the hTERT expression and subsequent telomerase activity were significantly enhanced. This result indicates that miR-17-92 is involved in the regulation of crosstalk between c-Myc/E2F1, and its upregulation can attenuate the restriction of E2F1 on c-Myc-induced hTERT expression, and as a result, boost the risk of tumorigenesis. In addition, we also hypothesized that mutants of the hTERT promoter region might be another mechanism by which it escapes from the transcriptional suppression of E2F1 and due to this fact, normal cells can easily be transformed by oncogenic c-Myc activation. Fig. 5 is a schematic diagram of how E2F1 negatively regulates c-Myc-induced hTERT transcription and the involvement of the miR-17-92 cluster (40).

Taken together, in the present study, we showed that E2F1 acts as a negative feedback regulator of c-Myc-induced hTERT transcription. If this negative regulating mechanism of E2F1 is attenuated or abrogated by miR-17-92 upregulation, hTERT is easily activated by c-Myc overexpression, thereby leading to cell immortalization and tumorigenesis.

\section{Acknowledgements}

This study was supported by the National Foundation of Natural Sciences, China (nos. 81101533, 81071727, 81170356 and 81270450) and China Postdoctoral Science Foundation (nos. 20100481468 and 201104755).

\section{References}

1. Wojtyla A, Gladych M and Rubis B: Human telomerase activity regulation. Mol Biol Rep 38: 3339-3349, 2011.

2. Harley CB: Telomerase and cancer therapeutics. Nat Rev Cancer 8: 167-179, 2008.

3. Kim NW, Piatyszek MA, Prowse KR, et al: Specific association of human telomerase activity with immortal cells and cancer. Science 266: 2011-2015, 1994.

4. Stewart SA and Weinberg RA: Telomeres: cancer to human aging. Annu Rev Cell Dev Biol 22: 531-557, 2006.

5. Land H, Parada LF and Weinberg RA: Tumorigenic conversion of primary embryo fibroblasts requires at least two cooperating oncogenes. Nature 304: 596-602, 1983.

6. Wu KJ, Grandori C, Amacker M, et al: Direct activation of TERT transcription by c-MYC. Nat Genet 21: 220-224, 1999.

7. Greenberg RA, O'Hagan RC, Deng H, et al: Telomerase reverse transcriptase gene is a direct target of c-Myc but is not functionally equivalent in cellular transformation. Oncogene 18: 1219-1226, 1999

8. Kyo S, Takakura M, Fujiwara T and Inoue M: Understanding and exploiting hTERT promoter regulation for diagnosis and treatment of human cancers. Cancer Sci 99: 1528-1538, 2008.

9. Matsumura I, Tanaka H and Kanakura Y: E2F1 and c-Myc in cell growth and death. Cell Cycle 2: 333-338, 2003.

10. Coller HA, Forman JJ and Legesse-Miller A: 'Myc'ed messages': myc induces transcription of E2F1 while inhibiting its translation via a microRNA polycistron. PLoS Genet 3: e146, 2007.

11. Crowe DL, Nguyen DC, Tsang KJ and Kyo S: E2F-1 represses transcription of the human telomerase reverse transcriptase gene. Nucleic Acids Res 29: 2789-2794, 2001.

12. Won J, Chang S, Oh S and Kim TK: Small-molecule-based identification of dynamic assembly of E2F-pocket protein-histone deacetylase complex for telomerase regulation in human cells. Proc Natl Acad Sci USA 101: 11328-11333, 2004.

13. Lacerte A, Korah J, Roy M, Yang XJ, Lemay S and Lebrun JJ: Transforming growth factor-beta inhibits telomerase through SMAD3 and E2F transcription factors. Cell Signal 20: 50-59, 2008.

14. Esquela-Kerscher A and Slack FJ: Oncomirs - microRNAs with a role in cancer. Nat Rev Cancer 6: 259-269, 2006.

15. O'Donnell KA, Wentzel EA, Zeller KI, Dang CV and Mendell JT: c-Myc-regulated microRNAs modulate E2F1 expression. Nature 435: 839-843, 2005. 
16. He L, Thomson JM, Hemann MT, et al: A microRNA polycistron as a potential human oncogene. Nature 435: 828-833, 2005.

17. Nagel S, Venturini L, Przybylski GK, et al: Activation of miR-17-92 by NK-like homeodomain proteins suppresses apoptosis via reduction of E2F1 in T-cell acute lymphoblastic leukemia. Leuk Lymphoma 50: 101-108, 2009.

18. Chang TC, Yu D, Lee YS, et al: Widespread microRNA repression by Myc contributes to tumorigenesis. Nat Genet 40 : 43-50, 2008.

19. Sylvestre Y, De Guire V, Querido E, et al: An E2F/miR-20a autoregulatory feedback loop. J Biol Chem 282: 2135-2143, 2007.

20. Li Y, Li Y, Zhang H and Chen Y: MicroRNA-mediated positive feedback loop and optimized bistable switch in a cancer network involving miR-17-92. PLoS One 6: e26302, 2011.

21. Wong JV, Yao G, Nevins JR and You L: Viral-mediated noisy gene expression reveals biphasic E2f1 response to MYC. Mol Cell 41: 275-285, 2011.

22. Aguda BD, Kim Y,Piper-Hunter MG, Friedman A and Marsh CB: MicroRNA regulation of a cancer network: consequences of the feedback loops involving miR-17-92, E2F, and Myc. Proc Nat Acad Sci USA 105: 19678-19683, 2008.

23. Liang GP, Luo XD and Yang ZC: Influence of human telomerase reverse transcriptase gene transfection on the proliferation of human embryonic fibroblasts. Zhonghua Shao Shang Za Zhi 21: 30-32, 2005 (In Chinese).

24. Yang SM, Fang DC, Yang JL, Chen L, Luo YH and Liang GP: Antisense human telomerase reverse transcriptase could partially reverse malignant phenotypes of gastric carcinoma cell line in vitro. Eur J Cancer Prev 17: 209-217, 2008.

25. Zhang YF, Li XH, Shi YQ, et al: CIAPIN1 confers multidrug resistance through up-regulation of MDR-1 and Bcl-L in $\mathrm{LoVo} / \mathrm{Adr}$ cells and is independent of p53. Oncol Rep 25 1091-1098, 2011

26. McNabb DS, Reed R and Marciniak RA: Dual luciferase assay system for rapid assessment of gene expression in Saccharomyces cerevisiae. Eukaryot Cell 4: 1539-1549, 2005.

27. Livak KJ and Schmittgen TD: Analysis of relative gene expression data using real-time quantitative PCR and the 2(-Delta Delta C(T)) method. Methods 25: 402-408, 2001.
28. Rounbehler RJ, Rogers PM, Conti CJ and Johnson DG: Inactivation of E2f1 enhances tumorigenesis in a Myc transgenic model. Cancer Res 62: 3276-3281, 2002.

29. Rounbehler RJ, Schneider-Broussard R, Conti CJ and Johnson DG: Myc lacks E2F1's ability to suppress skin carcinogenesis. Oncogene 20: 5341-5349, 2001.

30. Baudino TA, Maclean KH, Brennan J, et al: Myc-mediated proliferation and lymphomagenesis, but not apoptosis, are compromised by E2f1 loss. Mol Cell 11: 905-914, 2003.

31. Conner EA, Lemmer ER, Sánchez A, Factor VM and Thorgeirsson SS: E2F1 blocks and c-Myc accelerates hepatic ploidy in transgenic mouse models. Biochem Biophys Res Commun 302: 114-120, 2003.

32. Engelmann D and Pützer BM: The dark side of E2F1: in transit beyond apoptosis. Cancer Res 72: 571-575, 2012.

33. Polager S and Ginsberg D: p53 and E2f: partners in life and death. Nat Rev Cancer 9: 738-748, 2009.

34. Wu Z and Yu Q: E2F1-mediated apoptosis as a target of cancer therapy. Curr Mol Pharmacol 2: 149-160, 2009.

35. Leone G, Sears R, Huang E, et al: Myc requires distinct E2F activities to induce S phase and apoptosis. Mol Cell 8: 105-113, 2001.

36. Herold S, Herkert B and Eilers M: Facilitating replication under stress: an oncogenic function of MYC? Nat Rev Cancer 9: 441-444, 2009

37. Park JK, Chung YM, Kang S, et al: c-Myc exerts a protective function through ornithine decarboxylase against cellular insults. Mol Pharmacol 62: 1400-1408, 2002.

38. Lü MH, Liao ZL, Zhao XY, et al: hTERT-based therapy: A universal anticancer approach (Review). Oncol Rep 28 1945-1952, 2012

39. Olive V, Jiang I and He L: mir-17-92, a cluster of miRNAs in the midst of the cancer network. Int J Biochem Cell Biol 42: 1348-1354, 2010

40. Zhang Y, Fang D, Yang S and Fang D: E2F1: a potential negative regulator of hTERT transcription in normal cells upon activation of oncogenic c-Myc. Med Sci Monit 18: RA12-RA15, 2012. 